\title{
Automated Compositional Proofs for Real-Time Systems
}

\author{
Carlo A. Furia, Matteo Rossi, Dino Mandrioli, and Angelo Morzenti \\ Dipartimento di Elettronica e Informazione, \\ Politecnico di Milano, \\ 32, Piazza Leonardo da Vinci, 20133, Milano, Italy \\ \{furia, rossi, mandrioli, morzenti\}@elet.polimi.it
}

\begin{abstract}
We present a framework for formally proving that the composition of the behaviors of the different parts of a complex, real-time system ensures a desired global specification of the overall system. The framework is based on a simple compositional rely/guarantee circular inference rule, plus a small set of conditions concerning the integration of the different parts into a whole system. The reference specification language is the TRIO metric linear temporal logic.

The novelty of our approach with respect to existing compositional frameworks - most of which do not deal explicitly with real-time requirements - consists mainly in its generality and abstraction from any assumptions about the underlying computational model and from any semantic characterizations of the temporal logic language used in the specification. Moreover, the framework deals equally well with continuous and discrete time. It is supported by a tool, implemented on top of the proof-checker PVS, to perform deduction-based verification through theorem-proving of modular real-time axiom systems.

As an example of application, we show the verification of a real-time version of the old-fashioned but still relevant "benchmark" of the dining philosophers problem.
\end{abstract}

Keywords: Formal verification, modular systems, real-time, compositionality, rely/guarantee, axiom systems.

\section{Introduction}

Formal methods are more and more recognized to be a useful tool for the development of applications, as they allow their users to precisely verify the correctness of systems in their early development phases, before uncaught mistakes become overly costly to fix. One drawback often attributed to formal methods, however, is that they do not "scale up", i.e. when the system grows in complexity, they are too cumbersome and unwieldy to be used effectively. A natural solution

\footnotetext{
* Work supported by the MIUR project: "Quack: Piattaforma per la qualità di sistemi embedded integrati di nuova generazione."
} 
to this problem is to apply well-known software engineering principles such as modularity and separation of concerns to the verification of formal models. A compositional framework can help in this regard, in that it allows one to focus on the single parts of the system at first, and then analyze their mutual interactions at a later moment with a smaller effort than it would be required if all aspects (local and global) of the application were taken into account at once.

This paper presents a compositional inference rule for the TRIO language [5] that is suitable to formally prove the correctness of the behavior of a modular system from the behavior of its components. TRIO is a metric temporal logic for modeling and analysis of time-critical systems, and has been used in a number of industrial projects. Its advanced modular features are useful in writing specifications of complex systems. Our framework combines these features with the compositional inference rule through some application methodology that facilitates its practical use in structured specifications.

The approach followed in this paper belongs to the general framework of axiom systems, where a specification consists of a set of logic formulas and the verification consists in formally demonstrating that certain desired properties follow deductively from the specification formulas. This framework is indeed very general and abstract, as it does not rely on specific semantic assumptions and is independent of any notion of underlying computational model (to be considered when moving from specification towards implementation). In fact, proofs in our framework are to be intended as in classic logic deduction [15], and are supported and semi-automated by the theorem proving tool PVS [18]. Also, even if the reference language is TRIO, the results can be easily extended to any logic formalisms for the description of real-time axiom systems.

The paper is structured as follows: Section 2 shortly introduces the TRIO language; Section 3 presents a proof-oriented compositional framework for TRIO; Section 4 applies the framework to a timed version of Dijkstra's dining philosophers problem [8]; Section 5 reviews the most important compositional rules and frameworks in the literature, and points out where our approach differs from previous works on this subject; Section 6 draws conclusions and outlines future research.

For reasons of space, some of the formulas and proofs discussed in the paper have been omitted. The interested reader can find these details in an extended version of the paper, available online [11].

\section{TRIO}

TRIO [5] is a general-purpose specification language suitable to describe realtime systems. It is a first-order linear temporal logic that supports a metric on time. In addition to the usual propositional operators and quantifiers, it has a basic modal operator, called Dist, that relates the current time, which is left implicit in the formula, to another time instant: given a time-dependent formula $F$ (i.e. a term representing a mapping from the time domain to truth values) and a term $t$ indicating a time distance, the formula $\operatorname{Dist}(F, t)$ specifies that $F$ holds 
Table 1. TRIO derived temporal operators

\begin{tabular}{|c|c|}
\hline OPERATOR & DEFINITION \\
\hline $\operatorname{Past}(F, t)$ & $t \geq 0 \wedge \operatorname{Dist}(F,-t)$ \\
\hline$F u t r(F, t)$ & $t \geq 0 \wedge \operatorname{Dist}(F, t)$ \\
\hline $\operatorname{Som}(F)$ & $\exists d: \operatorname{Dist}(F, d)$ \\
\hline$A l w(F)$ & $\forall d: \operatorname{Dist}(F, d)$ \\
\hline $\operatorname{AlwP}(F)$ & $\forall d>0: \operatorname{Past}(F, d)$ \\
\hline$A l w F(F)$ & $\forall d>0: F u t r(F, d)$ \\
\hline Lasted $(F, t)$ & $\forall d \in(0, t): \operatorname{Past}(F, d)$ \\
\hline $\operatorname{Lasts}(F, t)$ & $\forall d \in(0, t): F u t r(F, d))$ \\
\hline$W i \operatorname{thin}(F, t)$ & $\exists d \in(0, t): \operatorname{Past}(F, d) \vee F u t r(F, d)$ \\
\hline$W i \operatorname{thin} P(F, t)$ & $\exists d \in(0, t): \operatorname{Past}(F, d)$ \\
\hline Within $F(F, t)$ & $\exists d \in(0, t): F u t r(F, d)$ \\
\hline Since $(F, G)$ & $\exists d>0: \operatorname{Lasted}(F, d) \wedge \operatorname{Past}(G, d)$ \\
\hline$U n t i l(F, G)$ & $\exists d>0: \operatorname{Lasts}(F, d) \wedge F u \operatorname{Fu}(G, d)$ \\
\hline$U p \operatorname{ToNow}(F)$ & $\begin{cases}\exists d>0: \text { Lasted }(F, d)) & \text { if dense } \\
\operatorname{Past}(F, 1) & \text { if discrete }\end{cases}$ \\
\hline NowOn $(F)$ & $\begin{cases}\exists d>0: \operatorname{Lasts}(F, d)) & \text { if dense } \\
F u \operatorname{tr}(F, 1) & \text { if discrete }\end{cases}$ \\
\hline Becomes $(F)$ & $\operatorname{UpToNow}(\neg F) \wedge(F \vee \operatorname{NowOn}(F))$ \\
\hline
\end{tabular}

at a time instant whose distance is exactly $t$ time units from the current instant. Notice that, in this paper, we deliberately do not formally specify a semantics for the interpretation of TRIO formulas: in fact all the discussion is independent of how the modal operator Dist is interpreted, and of which computational model is chosen, since it involves only syntactic manipulation of formulas.

A number of derived temporal operators can be defined from the basic Dist operator through propositional composition and first-order logic quantification. Table 1 shows those used in this paper. Notice that TRIO operators predicating on intervals by default do not include the interval boundaries. We define variations that may or may not include such boundaries by using the subscripts $i$ (included) or $e$ (excluded). E.g. $\operatorname{Alw} P_{i}(F) \equiv \forall d \geq 0: \operatorname{Past}(F, d)$, $\operatorname{AlwP}_{e}(F) \equiv \operatorname{AlwP}(F)$, WithinF $_{\text {ei }} \equiv \exists d \in(0, t]: F u \operatorname{Fr}(F, d)$, WithinF $_{i i} \equiv \exists d \in$ $[0, t]: F u t r(F, d)$, etc.

TRIO is well-suited to deal with both dense and discrete time. For specifying large and complex systems, and to support encapsulation, reuse and information hiding at the specification level, TRIO has the usual object-oriented constructs such as classes, inheritance and genericity. The basic encapsulation unit is the class, which is a collection of parameters, basic items, formulas and an interface.

Items are the primitive elements of the specification, such as predicates, timedependent variables, functions, etc. States and events are time-dependent items with a particular temporal behavior: events are predicates that are true only at isolated time instants; states are predicates which are true on non-empty time intervals (see [12] for a more precise definition).

We illustrate TRIO's features by introducing the specification of the dining philosophers problem [8]. Our solution is based on a philosopher class and assumes 
a continuous time model. Continuous time introduces some peculiar difficulties in the specification and verification phases, which we will handle exploiting an axiomatic-deductive approach. The basic items of the class are the event item start for the system initialization, and the items take $(s)$ and release $(s)$, with $s \in\{l, r\}$, indicating, for each philosopher, the action of taking or releasing the left or right fork. Other items are the state eating, which is true when the philosopher is eating, and the states holding $(s)$ and available $(s)$, meaning that the philosopher is holding a given fork or that the fork is available (i.e. not held by the adjacent philosopher). The philosopher class is parametric with respect to three constants $t_{e}, T_{e}, T_{t}$. They denote, respectively, the minimum eating time, the maximum eating time and the thinking time after an eating session, before becoming hungry again. Obviously, we assume $T_{e}>t_{e}>0$.

For each TRIO class, formulas are divided into three categories: axioms, assumptions and theorems. Axioms postulate the basic behavior of the system, assumptions express constraints we must discharge by means of other parts of the system (external to the current class) and theorems describe properties that are derived from other formulas. Any TRIO formula is implicitly universally quantified with the Alw operator.

We formalize the basic behavior of a philosopher through axiom formulas. For the sake of space limit, we do not present explicitly all the formulas of the example, but just give an informal presentation of some of them. The interested reader can find the complete example in the extended version of this paper available online [11]. We postulate that each philosopher always takes and releases both forks simultaneously (axiom holding_synch); consequently, if only one fork is available, the philosopher waits till the other fork becomes available as well. ${ }^{1}$ A philosopher becomes "hungry" when he/she has not eaten for a period longer than $T_{t}$. If he/she is hungry and if the forks are available, two situations are possible: either he/she takes both forks, or nondeterministically one of his/her neighbors takes a fork at that very time, so that the fork is not available anymore and the philosopher "loses his/her turn". This is formalized by axiom hungry.

Axiom 1 (hungry) Lasted( ( eating, $\left.T_{t}\right) \wedge U p T o N o w($ available $(l) \wedge$ available $(r))$ $\Rightarrow(\operatorname{take}(l) \wedge \operatorname{take}(r)) \vee N o w O n(\neg$ available $(l) \vee \neg$ available $(r))$.

Axioms eating_def and eating_duration state that, when a philosopher succeeds in acquiring both forks, he/she eats for a time duration of more than $t_{e}$ and less than $T_{e}$ time units, after which he/she releases both forks. Whenever a philosopher holds both forks we consider him/her eating. When he/she is not eating we say he/she is thinking (axiom thinking). Finally, a thinking session which has just begun lasts $T_{t}$ time units (axiom thinking_duration).

Each TRIO class has an interface, defined as the set of items and formulas that are externally visible. The user can declare each item to be visible or

${ }^{1}$ We introduce this simplification with respect to the traditional formulation because our example aims at proving a real-time, non starvation property, rather than the absence of deadlocks. 
non-visible; henceforth all the formulas predicating on visible items only are considered as visible, while all the other formulas are considered as non-visible outside the class. The interface is synthetically represented with a graphical notation: Figure 1 illustrates the interface of the philosopher class.

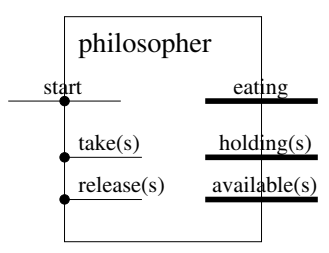

Fig. 1. Interface of the philosopher class

A structured TRIO specification is a collection of modules, i.e. instances of TRIO classes. The behavior of the overall composite system is given by the combination of the behaviors of its modules (i.e. it is defined by the logical conjunction of the axioms). We compose $N \geq 2$ instances of the philosopher class into the new composite class dining_N. The $N$ modules of the philosopher class are instantiated in an array Philosophers indexed by the range $[0 . . N-1]$. The modules are connected so that the available item of each philosopher corresponds to the negation of the holding item of the philosopher on his/her left/right.

\section{A Compositional Framework in TRIO}

In this section, we introduce a compositional framework for axiom systems, and the TRIO language in particular. The rationale of our approach is the following. The specification of a complex system is structured into classes. The fundamental behavior of each class is captured by axiom formulas. The derived behavior of each class can be expressed by theorem formulas. In general, according to the rely/guarantee paradigm, we want to relate the derived behavior of a class with certain properties of the (external) environment we assume to hold. When we compose the class with other classes constituting its actual environment, we have to discharge (i.e. prove) the assumption formulas by means of formulas of other classes. If the assumptions on the environment are temporally closed formulas (i.e. they express time-invariant properties), we may use TRIO assumption formulas to represent them; then, when discharging them, it is important to avoid circularities, in order to guarantee the soundness of the composite specification. If, on the other hand, we have to express assumptions on the environment that are directly temporally related with the derived behavior they guarantee, ${ }^{2}$ we need a new operator: the $\rightarrow$ operator (called time progression ) for the TRIO language, introduced in Section 3.2. When we compose the class with other classes,

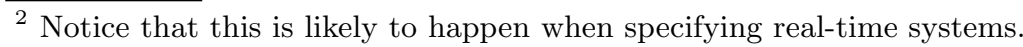


rely/guarantee formulas expressed using the $\rightarrow$ operator are composed using an ad hoc inference rule which handles temporal circularities correctly, according to the semantics of the operator. Finally, once the local assumptions have been discharged, we can infer the global specification from the logical composition of the (valid) local specifications.

Notice that the $\rightarrow$ operator, while similar to the operators presented in $[1,16]$, differs from them in that it does not impose any constraints nor conditions on the properties involved and the underlying computational model of the formalism. Section 5 discusses this issue in more detail.

\subsection{Rely/Guarantee Specifications}

Let us consider the rely/guarantee specification of a TRIO class $C$ written according to the following guideline. The basic behavior of $C$ is defined in terms of axioms over both visible and non visible items, which rely on no assumptions, since they just state the very basic behavior of the class. Then, we wish to derive a number of remarkable properties of the class as theorems. These theorems often depend on assumptions about the behavior of the environment. In TRIO, these assumptions can be stated using the language construct of the assumption formula. Let us name $\mathcal{A} \mathcal{X}_{C}, \mathcal{A S}_{C}$ and $\mathcal{T} \mathcal{H}_{C}$ the set of all axioms, assumptions and theorems of class $C$, respectively, and let $\mathcal{F}=\mathcal{A X}_{C} \cup \mathcal{A S}_{C} \cup \mathcal{T} \mathcal{H}_{C}$ be the set of all formulas of $C$. Furthermore, for each set of formulas $\mathcal{F}$, we define $\mathcal{F}^{\vee} \subseteq \mathcal{F}$ to be the set of visible formulas in $\mathcal{F}$, i.e. the formulas predicating over visible items only (see Section 2). Therefore, the complete specification of $C$ is represented by the formula $\mathcal{A X} \mathcal{X}_{C} \wedge \mathcal{A S}_{C} \Rightarrow \mathcal{T} \mathcal{H}_{C}$

Let us map these ideas on the philosophers example. Section 2 showed some axioms of the philosopher class. A derived property of the class we want to state is that there is always a time interval in which both forks are available to the philosopher. In our axiomatization, it suffices to show that this time interval ends at a time distant at most $T_{t}+2 T_{e}$ time units. The above property is expressed by the following TRIO formula.

\section{Theorem 2 (fork_availability)}

Within $F_{\text {ei }}(U p$ ToNow (available $(l) \wedge$ available $\left.(r)), T_{t}+2 T_{e}\right)$

The validity of this theorem cannot be guaranteed regardless of the behavior of the environment of this class. Therefore, we introduce three assumption formulas that suffice to deduce Theorem 2. First of all, each fork has to become available within $T_{t}+T_{e}$ time units or be already available and remain so for a sufficiently long (i.e. $\geq T_{t}$ ) amount of time. Second, we want each fork to be available, for a non-empty time interval, within $T_{e}$ time units. This is basically like assuming that the adjacent philosophers eat no longer than $T_{e}$ time units. Finally, when a fork becomes available, we assume it to stay so for (at least) $T_{t}$ time units, i.e. the thinking time of the neighbor philosophers is no shorter than $T_{t}$. These three assumptions are formalized by three formulas named availability, availability_2 and lasting_availability (not shown here for brevity). 
Let us now formalize what happens when composing TRIO classes. Let us consider $n$ modules $C_{1}, \ldots, C_{n}$. For all $i=1, \ldots, n$, module $C_{i}$ has a rely/guarantee specification expressed synthetically by the formula $\mathcal{A X}_{i} \wedge \mathcal{A} \mathcal{S}_{i} \Rightarrow \mathcal{T H}_{i}$. Let $C_{g l o b}$ be the class obtained by composing the $n$ instances $C_{i}$ as modules of $C_{\text {glob }}$. The composition of the $n$ modules is described by the logical conjunction of all the local specification formulas. Therefore TRIO classes are compositional, in that the semantics of the composition of classes is given by the logical conjunction of the semantics of the classes which are put together. In general, class $C_{g l o b}$ has its own axioms, assumptions and theorems, besides those of its modules, to allow the recursive application of the method. Hence, $C_{g l o b}$ is described by the formula $\mathcal{A X}_{\text {glob }} \wedge \mathcal{A S}_{\text {glob }} \Rightarrow \mathcal{T} \mathcal{H}_{\text {glob }}$.

We seek a way to prove:

$$
\mathcal{A X}_{\text {glob }} \wedge \mathcal{A S}_{\text {glob }} \wedge \bigwedge_{j=1, \ldots, n}\left(\mathcal{A X}_{j} \wedge \mathcal{A S}_{j} \Rightarrow \mathcal{T} \mathcal{H}_{j}\right) \Rightarrow \mathcal{T} \mathcal{H}_{\text {glob }}
$$

Hence, we want to find a way to discharge the local assumptions of each class by means of visible formulas of other classes, so that we can in turn use the validity of the local visible theorems to deduce global results. As it is simple to realize, this kind of reasoning involves a circularity between assumptions and guarantees of the modules, so that a naïve rule does not guarantee soundness, in general. We want to rule out these invalid reasonings in order to obtain a valid rule for composition. To this end we introduce a new temporal operator suitable to express rely/guarantee compositional specifications, and different conditions on the initial validity of the environment assumptions and on how to discharge them. Through these elements, we finally provide a valid compositional rule for rely/guarantee reasoning in TRIO.

\subsection{A Rely/Guarantee Inference Rule}

Let us introduce the $\rightarrow$ "time progression" operator for the TRIO language, suitable for expressing temporal relationships between assumptions and guarantees. Let $P$ and $Q$ be two time-dependent formulas. We define the $\rightarrow$ operator as a shorthand for the formula:

$$
P \rightarrow Q \triangleq\left\{\begin{array}{l}
\text { if dense }: A l w P_{e}(P) \Rightarrow A l w P_{i}(Q) \wedge \operatorname{NowOn}(Q) \\
\text { if discrete }: A l w P_{e}(P) \Rightarrow A l w P_{i}(Q)
\end{array}\right.
$$

Informally speaking, $P \rightarrow Q$ means that $Q$ lasts at least as long as $P$ does and even "a bit longer".

Now, we consider rely/guarantee specifications whose semantics is given in terms of the $\rightarrow$ operator. Therefore, if $E$ is the environment assumption and $M$ is the guarantee, the rely/guarantee specification is written as $E \rightarrow M$. Notice that we now admit temporally open formulas to be assumptions and guarantees.

Let us state, without proof, the following property of the $\rightarrow$ operator; the interested reader can find a proof in [11-Appendix A]. Note that the lemma holds both in dense and in discrete time models. 
Lemma 1. For any formulas $P, Q$ and $R$, if:

1. $\operatorname{Som}\left(\operatorname{Alw} P_{e}(P)\right)$

2. $A l w(Q \wedge R \Rightarrow P)$

then:

$$
A l w(P \rightarrow Q) \Rightarrow A l w(R \rightarrow Q)
$$

The following proposition states a sound inference rule $e^{3}$ for the $\rightarrow$ operator.

Proposition 1 (Rely/Guarantee Compositional Inference Rule). If, for $i=1, \ldots, m\left(m \in \mathbb{N}^{+}\right)$:

1. $\operatorname{Som}\left(\operatorname{Alw} P_{e}\left(E_{i}\right)\right)$ (that is $E_{i}$ is initialized)

2. $E \wedge \bigwedge_{j=1, \ldots, m} M_{j} \Rightarrow E_{i}$

3. $\wedge_{j=1, \ldots, m} M_{j} \Rightarrow M$

then: $\operatorname{Alw}\left(\bigwedge_{j=1, \ldots, m}\left(E_{j} \rightarrow M_{j}\right)\right) \Rightarrow \operatorname{Alw}(E \rightarrow M)$.

Proof. Assume $A l w\left(\bigwedge_{j=1, \ldots, m}\left(E_{j} \rightarrow M_{j}\right)\right)$. It is simple to realize, by considering the definition of the time progression operator, that this implies Alw $\left(\left(\bigwedge_{j=1, \ldots, m} E_{j}\right) \rightarrow\left(\bigwedge_{j=1, \ldots, m} M_{j}\right)\right)$. Moreover, hypothesis 2 implies that $A l w\left(E \wedge \bigwedge_{j=1, \ldots, m} M_{j} \Rightarrow \bigwedge_{j=1, \ldots, m} E_{j}\right)$, since it holds for every $i=1, \ldots, m$. Finally, hypothesis 1 implies that $\operatorname{Som}\left(\operatorname{Alw} P_{e}\left(\bigwedge_{=1, \ldots, m} E_{i}\right)\right)$, since there exists a base interval such that the conjunction of the $E_{i}$ 's is true on it ${ }^{4}$.

Therefore, we can apply Lemma 1 by substituting $\bigwedge_{j=1, \ldots, m} E_{j}$ for $P$, $\bigwedge_{j=1, \ldots, m} M_{j}$ for $Q$ and $E$ for $R$. We get:

$\operatorname{Alw}\left(\left(\bigwedge_{j=1, \ldots, m} E_{j}\right) \rightarrow\left(\bigwedge_{j=1, \ldots, m} M_{j}\right)\right) \Rightarrow \operatorname{Alw}\left(E \rightarrow\left(\bigwedge_{j=1, \ldots, m} M_{j}\right)\right)$.

Finally, by combining it with hypothesis 3 and with the definition of the time progression operator, we get the desired result.

Furthermore, we introduce a variation of the $\rightarrow$ operator for dense time domains: the $\rightarrow_{i}$ operator. $P \rightarrow_{i} Q$ is defined as $\operatorname{Alw} P_{i}(P) \Rightarrow \operatorname{Alw} P_{i}(Q) \wedge$ $\operatorname{NowOn}(Q)$, and Proposition 1 is valid for $\rightarrow_{i}$ as well. This variation will be used in Section 4.

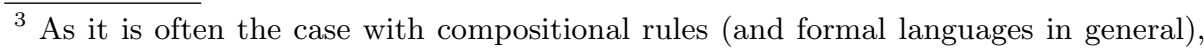
there is a trade off between (relative) completeness and simplicity and ease of use $[16,14]$. In this work, we have chosen to privilege the latter over the former, so that the inference rule in Proposition 1 is incomplete, as several other compositional rules in the literature $[16,4]$. For the sake of space limit, we do not discuss this issue in depth, leaving it for future extensions of this work.

${ }^{4}$ Consider the intersection of the intervals on which each of the $E_{i}$ 's is individually true; this intersection is non-empty, since all intersected intervals are unbounded on the left, because of the AlwP operator. 


\subsection{Integrating TRIO Modules}

Let us consider the composition of $n$ modules $C_{1}, \ldots, C_{n}$. We want to show briefly how the inference rule of Proposition 1 can be used in a large TRIO specification, integrating it with generic TRIO formulas. In general, each of the $n$ modules we compose may have one or more rely/guarantee formulas of the form $E \rightarrow M$ among its theorems. For each module $j=1, \ldots, n$, let $\mathcal{T H}_{j}^{r g} \subseteq \mathcal{T} \mathcal{H}_{j}$ be the set of theorems in the form $E \rightarrow M$ of class $j$. More formally, for each theorem formula $F \in \mathcal{T H}_{j}, F \in \mathcal{T} \mathcal{H}_{j}^{r g}$ if and only if $F$ can be written as $F \equiv E \rightarrow M$ for some formulas $E$ and $M$. Let us also define $m$ to be the number of such formulas over all classes: $m=\sum_{j=1, \ldots, n}\left|\mathcal{T} \mathcal{H}_{j}^{r g}\right|$. Moreover, $\mathcal{T} \mathcal{H}_{j}^{n r g}$ is defined as the complement set $\mathcal{T} \mathcal{H}_{j} \backslash \mathcal{T} \mathcal{H}_{j}^{r g}$ for all $j=1, \ldots, n$. The composite class $C_{\text {glob }}$ also has its own rely/guarantee formula $E_{g l o b} \rightarrow M_{\text {glob }}$ among its theorems $\mathcal{T H}_{\text {glob. }}$.

Now, we have to define what is a dependency between two formulas. Let us consider a formal proof $\pi$ : it consists of a finite sequence of formulas, together with their justifications (see, for example, [15]). We say that a formula $\chi$ directly depends upon another formula $\phi$ in the proof $\pi$, and write $\phi \rightsquigarrow_{\pi} \chi$, if and only if $\phi$ appears before $\chi$ in the proof and $\chi$ is the result of the application of an inference rule which uses $\phi$. The transitive closure $₫$ ("depends upon") of the $\rightsquigarrow$ relation $\phi \nrightarrow_{\pi} \chi$ is defined as usual. The notion of dependency can be extended to a set of proofs $\Pi$ : for any two formulas $\phi, \chi$ we say that $\phi \star^{\star} \Pi \chi$ if and only if there exists a proof $\pi \in \Pi$ such that $\phi \stackrel{\star}{\star} \chi$.

Finally, we can formulate a "checklist" to follow when verifying our composite specification. The rationale is that we avoid circularities in discharging temporally closed assumptions, and we resolve possible circularities between $\rightarrow$ specifications by using the inference rule of Proposition 1. More precisely, one should proceed as follows.

1. Verify each local specification, that is prove that for all $k=1, \ldots, n: \mathcal{A X}_{k} \wedge$ $\mathcal{A S}_{k} \Rightarrow \mathcal{T} \mathcal{H}_{k}$. From our perspective, this step is considered to be atomic, but obviously the compositional approach can be applied recursively to each module.

2. Show that the local assumptions can be discharged by means of global formulas, local axioms and theorems, and visible formulas of other classes. In formulas, this corresponds to proving that for all $k=1, \ldots, n: \mathcal{F}_{\text {glob }} \wedge \mathcal{A X}_{k} \wedge$ $\mathcal{T} \mathcal{H}_{k} \wedge \bigwedge_{j=1, \ldots, n \wedge j \neq k} \mathcal{F}_{j}^{\vee} \Rightarrow \mathcal{A S}_{k}$.

3. Prove that the global non-rely/guarantee theorems (i.e. not involving the $\rightarrow$ operator) follow from the local visible formulas and from the global axioms, assumptions and other (i.e. rely/guarantee) theorems. In formulas, this means proving $\mathcal{A X}_{\text {glob }} \wedge \mathcal{A S}_{\text {glob }} \wedge \mathcal{T} \mathcal{H}_{\text {glob }}^{r g} \wedge \bigwedge_{j=1, \ldots, n} \mathcal{F}_{j}^{\vee} \Rightarrow \mathcal{T} \mathcal{H}_{\text {glob }}^{\text {nrg }}$.

4. Show that each local rely/guarantee formula has an assumption which satisfies the initialization condition (as in hypothesis 1 of Proposition 1). In order to prove the initialization condition, we can use global and local formulas, plus any visible formula of any other class of the system. In formulas, this corresponds to proving that for all $k=1, \ldots, m$ : for all $j=1, \ldots, n$ : if $\left(E_{k} \rightarrow M_{k}\right) \in \mathcal{T H}_{j}^{r g}$ then $\mathcal{F}_{\text {glob }} \wedge \mathcal{F}_{j} \wedge \bigwedge_{i=1, \ldots, n} \mathcal{F}_{i}^{\vee} \Rightarrow \operatorname{Som}\left(\operatorname{Alw} P_{e}\left(E_{k}\right)\right)$. 
5. Show that each local rely/guarantee formula has an assumption that can be discharged by means of global and local formulas, or by the global assumption, or by means of guarantees of other classes. This corresponds to hypothesis 2 in Proposition 1. In formulas, this is proving that for all $k=1, \ldots, m$ : for all $j=1, \ldots, n$ : if $\left(E_{k} \rightarrow M_{k}\right) \in \mathcal{T H}_{j}^{r g}$ then: $\mathcal{F}_{\text {glob }} \wedge \mathcal{F}_{j} \wedge \bigwedge_{i=1, \ldots, n} \mathcal{F}_{i}^{\underline{v}} \Rightarrow$ $\operatorname{Alw}\left(E_{g l o b} \wedge \bigwedge_{i=1, \ldots, m} M_{i} \Rightarrow E_{k}\right)$.

6. Show that the global guarantee follows from the local guarantees of all modules and from global formulas and local visible formulas of any class. This corresponds to hypothesis 3 of Proposition 1. In formulas, this is proving $\mathcal{F}_{\text {glob }} \wedge \bigwedge_{j=1, \ldots, n} \mathcal{F}_{j}^{\underline{\mathrm{v}}} \Rightarrow \operatorname{Alw}\left(\bigwedge_{j=1, \ldots, m} M_{j} \Rightarrow M_{\text {glob }}\right)$.

7. Be sure that in all the above proofs there are no circular dependencies among any two closed formulas. Formally, this corresponds to checking that in the set $\Pi$ of all the above proofs, for all formulas $\phi \in \bigcup_{k=1, \ldots, n}\left(\mathcal{A S}_{k} \cup \mathcal{T} \mathcal{H}_{k}\right) \cup$ $\mathcal{T} \mathcal{H}_{\text {glob }}: \neg(\phi \pitchfork \Pi \phi)$.

From the application of the above steps, thanks to the inference rule of Proposition 1 and the absence of circularities, we conclude the validity of the global specification $\mathcal{A X}_{\text {glob }} \wedge \mathcal{A S}_{\text {glob }} \Rightarrow \mathcal{T} \mathcal{H}_{\text {glob }}$

\section{Compositional Dining Philosophers}

This section illustrates an analysis of the dining philosophers problem, with the compositional proofs of some relevant properties, as an example of compositional specification and verification in TRIO using the rely/guarantee paradigm. Even if the example does not constitute an "industrial-strength in-the-large" case study, we believe that, after several decades of successful application, it is still an insightful and thought-provoking example to assess the validity of - not only - our compositional rule in the short space of a conference paper. All details of the proofs have been checked with the encoding of the TRIO language in the PVS proof checker [18] (see [12,10] for some details of this encoding), even if we present them succinctly (due to space limit) and in human-readable form.

\subsection{One Rely/Guarantee Philosopher}

Assumptions availability, availability_2 and lasting_availability of Section 3.1 express the suppositions that each philosopher makes about the behavior of his/her neighbors. In turn, the philosopher must guarantee to them that he/she will not be unfair and will periodically release the forks. This requirement is expressed by two theorems, taking_turns and taking_turns_2 (not explicitly shown), that are analogous to the assumptions availability and availability_2, while assumption lasting_availability corresponds to axiom thinking_duration (see Section 2). For the sake of brevity, the proofs of these two theorems, which are directly derivable from the axioms of the philosopher class only, are not discussed here.

The local non-starvation property requires that, assuming a regular availability of the forks, we can guarantee that, after the system starts, the philosopher eats regularly. This requirement can be formalized using the $\rightarrow_{i}$ operator, relating the availability of the forks in the past to the occurrence of the eating sessions 
in the immediate future. In our case, $E_{k}=$ Within $F_{e i}(U p T o N o w$ (available $(l) \wedge$ available $\left.(r)), T_{t}+2 T_{e}\right)$ and $M_{k}=\operatorname{SomP}_{i}$ (start) $\Rightarrow\left(\exists t>t_{e}:\right.$ Within $_{i i}$ (Lasts (eating, $\left.\left.t), T_{t}+2 T_{e}\right)\right)$. The following theorem expresses the local non-starvation property, whose proof we omit for brevity. Notice that the proof assumes that the thinking time of each philosopher is larger than twice the eating time: $T_{t}>2 T_{e}$. After all, they are philosophers, not gourmands! (Unless they are Epicureans, one may argue...). This condition allows to avoid the race conditions.

\section{Theorem 3 (regular_eatings_rg) .}

$W_{\text {ithin }} F_{\text {ei }}(U p$ ToNow (available $(l) \wedge$ available $\left.(r)), T_{t}+2 T_{e}\right)$

$\rightarrow_{i} \quad\left(\operatorname{SomP}_{i}(\right.$ start $) \Rightarrow\left(\exists t>t_{e}: W_{i t h i n}\left(\operatorname{Lasts}_{i i}(\right.\right.$ eating, $\left.\left.\left.t), T_{t}+2 T_{e}\right)\right)\right)$

Up to this point, we have proved that $\mathcal{A} \mathcal{X}_{\text {phil }} \wedge \mathcal{A S}_{\text {phil }} \Rightarrow \mathcal{T H}_{\text {phil }}$, which corresponds to step 1 of Section 3.3.

\subsection{A Table of Philosophers}

The global non-starvation property is expressed by theorem liveness_rg below. It simply states that each philosopher in the array eats regularly, unless he/she has not started yet. Notice that in our example $E_{g l o b}=$ true since the composite system is closed, and $M_{g l o b}$ coincides with the following liveness_rg theorem.

Theorem 4 (liveness_rg) $\operatorname{SomP}_{i}($ Philosophers[i].start) $\Rightarrow$

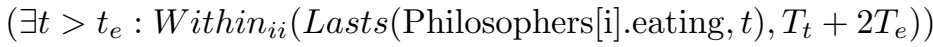

In Section 4.1 above, we completed step 1 of Section 3.3. Now, let us consider step 2. Each local assumption is discharged by either a global assumption or by a visible theorem or axiom of the modules adjacent to the current philosopher. Therefore, step 2 is completed without circularities involved, since thinking_duration is an axiom and taking_turns[_2] are both proved directly from axioms local to each class.

Step 3 is empty in our example, since the only global theorem we want to prove is theorem liveness_rg in $\mathcal{T H}_{\text {dining_N }}^{r g}$. Let us consider step 4, where we have to prove that each environment assumption $E_{k}$ is initialized, that is we have to show that hypothesis 1 of Proposition 1 holds. Since the local theorems have already been proved without circularities, we can use fork_availability to complete this step. The theorem simply states that the desired property $E_{k}=$ Within $F_{e i}\left(U p T o N o w(\right.$ available $(l) \wedge$ available $\left.(r)), T_{t}+2 T_{e}\right)$ always holds, which subsumes the initialization condition. Step 5 requires to discharge the $E_{k}$ 's by means of other formulas, in order to fulfill hypothesis 2 of Proposition 1 . Once again, the theorem fork_availability for class $k$ works correctly since it predicates the validity of the $E_{k}$ 's over the whole temporal axis. Step 6 is also very simple, since $M=\forall k \in\{1, \ldots, n\}: M_{k}$, so that the implication of this step holds trivially. As a consequence, hypothesis 3 of Proposition 1 is shown to hold.

As discussed above, no circularities arise in proving the local formulas, so we conclude that theorem liveness_rg holds as a consequence of the inference rule of Proposition 1 and following the steps in Section 3.3. 


\subsection{Complexity of the Proofs}

Let us briefly evaluate the complexity of the global proof outlined above, using the proof checker PVS. The PVS proofs of the dining_N system required a total of 926 prover commands; approximately half of them where devoted to the proof of the theorem regular_eatings_rg. Let us compare the cost of this verification with the cost of a non-compositional one.

The basic problem with a non-compositional proof is that we cannot exploit encapsulation and reuse. Therefore, there is no distinction between local and global items and everything is "flattened" at the same level of visibility. In the case of the dining philosopher problem, we can overcome this problem by "simulating" modularization at the global level. In other words, we have to carefully parametrize each item with respect to an index which separates different "instances" of the philosopher. Moreover, and most importantly, we must devise a way to replace the use of the $\rightarrow$ operator by temporally closed formulas only. This unstructured solution has been implemented; the result has been a proof of length comparable to the compositional one, but fragmented into more intermediate lemmas, with more assumptions and more intricate proof dependencies. Another feature that distinguishes the compositional proof from the non-compositional one is the fact that the former is repetitive while the latter is intricate. In other words, the compositional proof has a structure made of several similar parts, indicating that it is indeed simpler to manage for the human user who can easily understand when previous proof patterns can be applied again with minor modifications. All in all, even if the number of proof commands where not dramatically different in the two proofs, the complexity of the non-compositional one, considering also the mental effort and difficulty in managing the proof, was much greater. Furthermore, our experience with the compositional proof of the same property has guided and helped the building of the non-compositional one: we believe that doing the non-compositional proof first would have been really hard and time-consuming.

\section{$5 \quad$ Related Works}

A compositional analysis technique applies some, possibly formal, method to infer global properties of a large, complex system through a hierarchical and iterative process that exploits the system's modular structure. A general (and historical) introduction to compositional methods can be found in $[6,7]$. Without aiming at exhaustiveness, this section briefly reviews some of the most important contributions about compositional reasoning and shows how the approach of this paper differs from them.

An issue still largely unexplored in the present literature on compositionality is the consideration of hard real-time aspects, which require a metric modeling of time. A first noticeable exception is Ostroff in [17], where the metric temporal logic RTTL is embedded in a compositional framework. Nonetheless, the approach is rather different from ours, being focused on refinement aspects rather than on a posteriori composition that exploits reuse. Furthermore, time 
is treated as a separate variable and is discrete, while in our approach time is an implicit item of the language and can be either continuous or discrete.

The need for compositionality has become indisputable in the formal methods community, so that almost every newly introduced formalism encompasses some sort of compositional technique or permits compositional specifications. However, to the best of our knowledge, all proposed compositional frameworks are deeply rooted on some particular, often restrictive semantic assumptions, and depend explicitly on the underlying computational model. In this regard, formalisms typically assume either an interleaving semantics (e.g. $[1,9,16])$ or a synchronous semantics (e.g. [3]) for the concurrent components of the system.

A rather different compositional framework to support the top-down development of real-time systems based on logical formulas at the semantic level is studied by Hooman [13]. In a sense, Hooman's framework is independent of semantic assumptions, even if its set-theoretic model of semantic primitives naturally relates to interleaving semantics models. However, the framework is focused on the refinement (i.e. decomposition) aspect and basically consists of an inference rule that permits to deduce that the decomposition of a module into its refined parts correctly implements the original (unrefined) module. Another important difference between Hooman's framework and ours is that the former does not adopt the rely/guarantee paradigm, and is therefore suitable only to write specifications of modules who do not rely on a constrained behavior of the environment to function correctly.

More typical solutions to the problem of formulating a sound rely/guarantee compositional rule involve the use of an ad hoc operator to write rely/guarantee specifications so that they satisfy certain specific characterizations.

For example, the above approach is followed by Abadi and Lamport [1], who analyze the rely/guarantee compositional paradigm using TLA as the reference specification language. The authors introduce the TLA operator $\stackrel{t}{\rightarrow}$ to write rely/guarantee specifications that can be soundly composed. Notice that our paper also introduced a suitable operator (the time progression operator $\rightarrow$ ) to write rely/guarantee specifications. The crucial difference is that our time progression operator is applied in inference rules independently of any assumption on the semantics of processes and also of any semantic characterization of formulas (its application does not need notions such as safety, closure, etc., which are instead integral part of (among others) Abadi and Lamport's framework). This renders our framework purely syntactic and very general. In particular, even if the inference rule of [1] is usable for general properties, the conditions of the rule are hard to prove if they are not safety properties; such a distinction does not apply to a syntactic rule such as ours.

Abadi and Merz [2] propose an abstract generalization of rely/guarantee inference rules, in an attempt to treat compositionality syntactically. To this extent, a modal operator to write rely/guarantee inference rules is introduced with minimal semantic assumptions. However, the use of the operator in inference rules and the consequent soundness proofs are possible only after the abstract framework is specialized by choosing a semantic model and a computational 
model. On the contrary, in our framework the soundness of the inference rule is completely proved without assumptions of this kind.

Amla et al. [4] present an abstract compositional framework which can be considered as a generalization of several concrete compositional frameworks in the literature. In particular, they succeed in formulating an inference rule which does not rely on an ad hoc operator to be sound, and is therefore simpler than others. However, their framework still relies heavily on semantic assumptions, such as downward closure, on the set of behaviors describing a process. Therefore, our framework does not fit the models in [4], since it pursues the alternative (and new) approach of using a rely/guarantee operator, but independently of any semantic assumptions on the behavior of the components of the system, according to the axiomatic approach.

\section{Conclusions}

We presented a compositional framework for the TRIO specification language that supports verification through automated theorem proving. The framework is based on a formal notion of composition of TRIO modules, which is used to prove that the mutual interactions between components of a complex system guarantee some property for the global application, after the components are integrated into the system. The compositional rule has been proved sound and has been applied to the classic example of Dijkstra's dining philosophers as a simple, but not simplistic, example. The compositional framework has been encoded into the logic of the PVS theorem prover.

With respect to other approaches to compositionality in formal methods, our own emerges as more suitable for real-time modeling, it encompasses both continuous and discrete time to better model physical processes, and it is conceived for axiom systems and deductive verification. Therefore, the approach is very general and abstracts away from specific assumptions about process semantics and the underlying computational model.

Future work in this line of research will follow three main directions. First, the framework presented here is being applied to several real-life industrial case studies to experimentally evaluate its effectiveness. Second, alternative weaker or stronger - inference rules will be investigated. In particular, we are exploring variations and generalizations of the $\rightarrow$ operator, better suited to be applied on certain classes of systems, different inference rules which do not use a time progression operator at all, and complete inference rules (which sacrifice some simplicity of application). Third, automated support for the framework will be improved and extended.

\section{Acknowledgements}

The authors thank the anonymous reviewers for their remarks. 


\section{References}

1. M. Abadi and L. Lamport. Conjoining specifications. ACM TOPLAS, 17(3):507535, 1995.

2. M. Abadi and S. Merz. An abstract account of composition. In MFCS'95, volume 969 of $L N C S$, pages 499-508, 1995.

3. R. Alur and T. A. Henzinger. Reactive modules. Formal Methods in System Design, 15(1):7-48, 1999.

4. N. Amla, E. A. Emerson, K. S. Namjoshi, and R. J. Trefler. Abstract patterns of compositional reasoning. In CONCUR'03, volume 2761 of $L N C S$, pages 431-445, 2003.

5. E. Ciapessoni, A. Coen-Porisini, E. Crivelli, D. Mandrioli, P. Mirandola, and A. Morzenti. From formal models to formally-based methods: an industrial experience. ACM TOSEM, 8(1):79-113, 1999.

6. W.-P. de Roever. The need for compositional proof systems: a survey. In COMPOS'97, volume 1536 of $L N C S$, pages 1-22, 1998.

7. W.-P. de Roever, F. de Boer, U. Hannemann, J. Hooman, Y. Lakhnech, M. Poel, and J. Zwiers. Concurrency Verification: Introduction to Compositional and Noncompositional Methods. Cambridge University Press, 2001.

8. E. W. Dijkstra. Hierarchical ordering of sequential processes. In Operating Sys. Tech., pages 72-93. 1972.

9. B. Finkbeiner, Z. Manna, and H. B. Sipma. Deductive verification of modular systems. In COMPOS'97, volume 1536 of LNCS, pages 239-275, 1998.

10. C. A. Furia. Compositional proofs for real-time modular systems. Laurea degree thesis, Politecnico di Milano, 2003.

11. C. A. Furia, M. Rossi, D. Mandrioli, and A. Morzenti. Automated compositional proofs for real-time systems. Full version with appendices available online from http://www.elet.polimi.it/upload/furia, 2005.

12. A. Gargantini and A. Morzenti. Automated deductive requirement analysis of critical systems. ACM TOSEM, 10(3):255-307, July 2001.

13. J. Hooman. Compositional verification of real-time applications. In COMPOS'9'7, volume 1536 of $L N C S$, pages 276-300, 1998.

14. P. Maier. Compositional circular assume-guarantee rules cannot be sound and complete. In FOSSACS'03, volume 2620 of $L N C S$, pages 343-357, 2003.

15. E. Mendelson. Introduction to Mathematical Logic. Chapman \& Hall, 1997.

16. K. S. Namjoshi and R. J. Trefler. On the completeness of compositional reasoning. In $C A V^{\prime} 00$, volume 1855 of $L N C S$, pages 139-153, 2000.

17. J. S. Ostroff. Composition and refinement of discrete real-time systems. $A C M$ TOSEM, 8(1):1-48, 1999.

18. S. Owre, J. M. Rushby, and N. Shankar. PVS: A Prototype Verification System. In $C A D E-11$, volume 607 of $L N C S$, pages 748-752, 1992. 